\title{
Reduction of Propagation Loss in Pillar-Based Photonic Crystal Waveguides
}

\author{
Abigaël A. M. Kok, Member, IEEE, Jos J. G. M. van der Tol, Roel Baets, Fellow, IEEE, and \\ Meint K. Smit, Fellow, IEEE
}

\begin{abstract}
Out-of-plane losses are the major issue in the integration of two-dimensional photonic crystal devices in photonic integrated circuits. In this paper, we show that the out-of-plane losses of pillar-based photonic crystal waveguides can be vastly reduced, even for pillars with a low vertical index contrast, such as in InP/InGaAsP/InP technology. These low losses are obtained by creating confinement between the pillars with a polymer layer stack. We show that the spatial frequency component of the Bloch mode in the first Brillouin zone (i.e., the component inside the light cone), is significantly suppressed by the optimized polymer layer stack.
\end{abstract}

Index Terms-Bloch modes, out-of-plane loss, pillar-based photonic crystals.

\section{INTRODUCTION}

$\mathbf{T}$ WO-DIMENSIONAL photonic crystals (PhCs) have been subject to extensive research in the past years, since they offer a promising potential for integration in photonic integrated circuits (PICs). They can be used to miniaturize existing integrated optical devices, e.g., bends, microcavities, add-drop filters, and band edge lasers [1]-[3]. Furthermore, their special properties can be used to design devices based on new principles. Examples of this type of devices are polarization splitters and wavelength division multiplexers [4], [5]. The main issue for integration of PhC-based devices in PICs are the losses; those of $\mathrm{PhC}$ devices should be comparable or smaller than those of classical waveguide devices to be able to compete with them.

Photonic integrated circuits can be realized in different material systems, depending on the applications. However, indium phosphide (InP) technology is the only platform to monolithically integrate active and passive devices for use in the near-infrared wavelength region $(1300-1550 \mathrm{~nm})$, which is the most important range for telecom applications. The classical waveguides of InP PICs are based on total internal reflection; light is confined to the indium gallium arsenide phosphide (InGaAsP) core, which has a higher refractive index than the InP claddings. In two-dimensional $\mathrm{PhCs}$, the in-plane confinement is created by the photonic band gap properties of the crystal, whereas the light is confined out-of-plane by total internal reflection.

Manuscript received September 04, 2007; revised October 17, 2007. First published April 28, 2009; current version published August 18, 2009. This work was supported by NanoNed, a technology program of the Dutch Ministry of Economic Affairs via the foundation STW.

A. A. M. Kok, J. J. G. M. van der Tol, and M. K. Smit are with the COBRA Research Institute, Eindhoven University of Technology, Eindhoven 5600 MB, The Netherlands (e-mail: a.a.m.kok@tue.nl).

R. Baets is with the Ghent University-IMEC, Department of Information Technology, 9000 Gent, Belgium.

Digital Object Identifier 10.1109/JLT.2009.2020892
We can distinguish 2-D PhCs by their out-of-plane index contrast. Membrane-type photonic crystals-with the semiconductor layers sandwiched between dielectric layers or air-have a high refractive index contrast (e.g., freestanding InGaAsP membranes), whereas structures based on semiconductor layers only-often referred to as substrate-type stuctures-have a relatively small refractive index contrast. Furthermore, a 2-D $\mathrm{PhC}$ can either consist of etched (low-refractive-index) holes or of high-refractive-index rods. These distinctions lead to four possible geometries. One of the geometries, i.e., pillars with a high vertical index contrast, is not compatible with the classical PICs in InP technology. In recent years, the hole-type $\mathrm{PhCs}$ have been investigated by many research groups, both the membrane PhCs with high vertical index contrast [6] as well as the deeply etched holes with low vertical index contrast [7]. Despite of all the experimental research on hole-type $\mathrm{PhCs}$ in InP technology, no experiments have been published on InP-based pillar PhCs. That is most probably due to the common belief that these crystals have excessively high losses.

In this paper, we study pillar-based photonic crystal waveguides that are to be integrated in classical PICs on an InP substrate with a 500-nm-thick InGaAsP core layer and a 1- $\mu \mathrm{m}$-thick InP top cladding. A square lattice of high-index pillars exhibits a large TM band gap. Pillar PhCs have several advantages. To start with, the layer stack of pillar PhCs is compatible with that of the classical PIC, and so is the fabrication technology. Second, a single line defect in a pillar-based $\mathrm{PhC}$ supports one even symmetry mode which allows for effective single mode operation. Finally, active pillar PhC devices can be electrically contacted, inherently avoiding current spreading, and heat is efficiently dissipated to the substrate. To reduce the out-of-plane losses, we implement a polymer layer stack between the semiconductor pillars as is schematically shown in Fig. 1. We will show that after the implementation of the optimized polymer layer stack, pillar-based photonic crystals have extremely low propagation loss. Additionally, one could anticipate the use of functional polymers, such as polymers containing liquid crystals or nonlinear polymers, for various switching applications. Furthermore, the applied polymers can passivate active $\mathrm{PhC}$ pillars, thus reducing the nonradiative recombination at the surface of the active area.

To design and model the 2-D PhC components, 3-D calculations are needed. However, these calculations are very time consuming and impose strong requirements on computational power. Therefore the in-plane and out-of-plane confinement problems are treated separately in the present paper. In Section II, we focus on the in-plane confinement of the light and we describe the design of the pillar photonic crystal waveguide. 


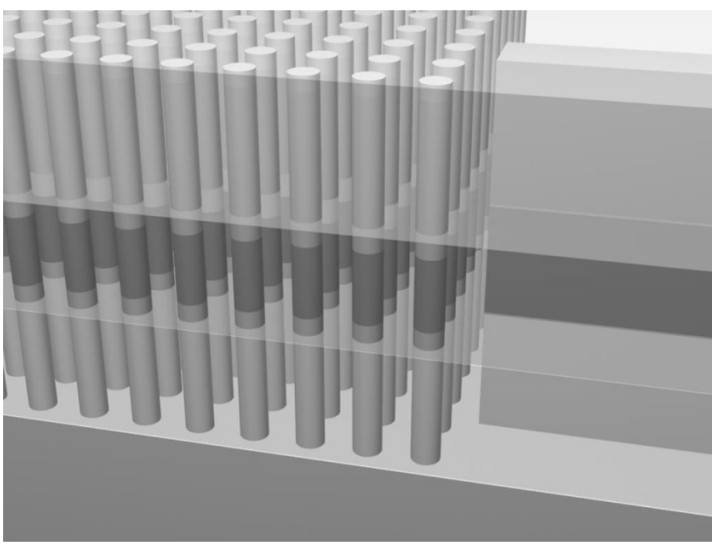

Fig. 1. Schematic representation of a classical ridge waveguide (right) and a pillar photonic crystal (left) with a polymer layer stack to create out-of-plane confinement between the pillars.

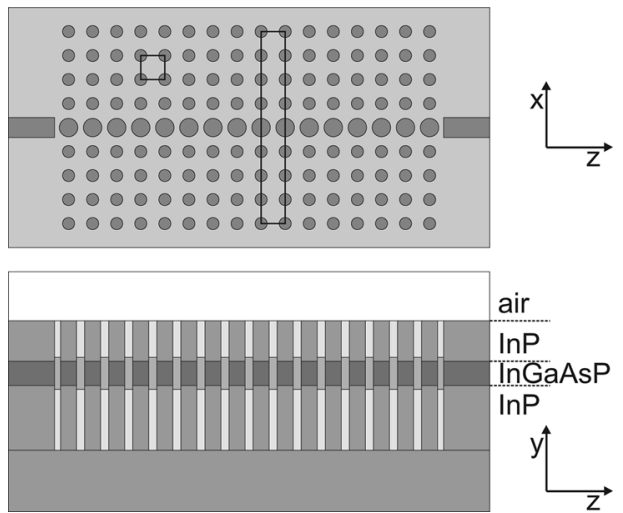

Fig. 2. Schematic representations of the pillar photonic crystal waveguide. The upper picture shows a top view, with the unit cells used in the 2-D band solver calculations indicated. The left unit cell is used to compute the band diagram of the undisturbed lattice, whereas the right super cell is used to compute the bands of the defect modes of the $\mathrm{PhC}$ waveguide. The lower picture shows a vertical cross section of the photonic crystal waveguide through the symmetry axis of the waveguide.

Section III handles the out-of-plane confinement. The polymer layer stack is optimized and we explain the physical mechanism that is responsible for the very low propagation loss.

\section{IN-PLANE CONFINEMENT}

All the simulations in this section are performed using a 2-D band solver [8], where the effective index method is used to account for the vertical dimension. Fig. 2 shows the structure that is used in the band solver calculations. The effective index of the fundamental guided TM mode of the semiconductor stack is calculated to be 3.2641. For pillars in air, the surrounding medium has a refractive index 1.0. The effective index of the optimized polymer layer stack is 1.5236 (to be discussed in Section III).

To start with, we optimized the ratio of radius over lattice constant $(r / a)$ of the bulk $\mathrm{PhC}$ lattice to get the largest photonic band gap. When a polymer layer stack is introduced between the pillars, the lateral index contrast is reduced. This implies that $\mathrm{n}$ case has the most stringent conditions to the photonic crystal lattice in order to open up a band gap. For the pillars in a polymer stack, the largest band gap is obtained if $r / a=0.25$.
The TM band gap ranges from $0.258(a / \lambda)$ to $0.294(a / \lambda)$. The lattice constant of a photonic crystal with the center frequency corresponding to $\lambda=1550 \mathrm{~nm}$ should be $a=\lambda \omega_{\mathrm{c}}=1550$. $0.276 \simeq 427 \mathrm{~nm}$ and the radius $r=0.25 a \simeq 107 \mathrm{~nm}$. The TM band gap ranges from $\lambda_{\min }=a / \omega_{\max } \simeq 1452 \mathrm{~nm}$ to $\lambda_{\max }=a / \omega_{\min } \simeq 1655 \mathrm{~nm}$.

If we take pillars in air with the same radius over lattice constant ratio $r / a=0.25$, the band gap size is increased due to the higher lateral index contrast. The TM band gap spans the normalized frequency range from $0.265(a / \lambda)$ to $0.368(a / \lambda)$. The center frequency of the TM band gap is $\omega_{\mathrm{c}}=0.317(a / \lambda)$ and the corresponding lattice constant is given by $a=\lambda \omega_{\mathrm{c}} \simeq 491 \mathrm{~nm}$. The corresponding pillar radius is $r=0.25 a \simeq 123 \mathrm{~nm}$. The stop band ranges from $\lambda_{\min }=a / \omega_{\max } \simeq 1334 \mathrm{~nm}$ to $\lambda_{\max }=a / \omega_{\min } \simeq 1853 \mathrm{~nm}$. For both pillars in air as well as pillars in a polymer stack, the photonic band gap is large enough for the intended applications operating in the EDFA window (1530-1570 nm).

By adding defects to photonic crystals, one can create in-plane confinement of the light [9]. A waveguide can be created in a planar photonic crystal by changing either the refractive index or the radius of a row of pillars in the bulk lattice. Since we are bound to the material stack of the PIC, the only practical possibility to tailor the crystal is to vary the pillar radius. We increase the radius of the line defect pillars, since larger pillars are more stable and have a higher effective refractive index. The 2-D band diagram of a $\mathrm{PhC}$ waveguide is calculated by using the band solver for a supercell, i.e., a unit cell that spans the waveguide defect and several lattice periods in perpendicular direction as shown in Fig. 2. The computed bands are projected onto the $\Gamma X$-direction, i.e., the propagation direction along the $\mathrm{PhC}$ waveguide. In this way, we find the Bloch modes that are allowed to propagate along the waveguide, but that are confined in-plane by the surrounding crystal.

The band diagram is calculated for varying defect radius $r_{\mathrm{d}}$ and the waveguide modes are evaluated on several aspects. First, the group velocity $v_{\mathrm{g}}=d \omega / d k$ of a waveguide mode should be large and constant over a reasonable bandwidth. Second, we aim for the excitation of even modes, having a field amplitude that has mirror symmetry with respect to the $y z$-plane through the centers of the pillars.

Fig. 3 shows the projected band diagram of the lattice of pillars in a polymer layer stack. For this $\mathrm{PhC}$ waveguide, the optimized radius of the defect pillars is $r_{\mathrm{d}}=155 \mathrm{~nm}$. There are two waveguide modes in the band gap between the first and the second band of the bulk lattice. The mode with monotonically increasing frequency is an odd mode, whereas the mode that is monotonically decreasing in frequency is an even mode.

The bulk photonic crystal was designed to have its center frequency corresponding to the wavelength $\lambda=1550 \mathrm{~nm}$, as represented in the band diagram by the dash-dotted line. At this frequency, the slope of the odd mode is close to zero, implying that the group velocity is very small. This means that the odd mode is in the so-called slow-light regime, where it propagates extremely slowly. At the crossing of the even and the odd mode, the waveguide modes become hybrid and a mini stop band appears. Excitation at this frequency should be avoided. 


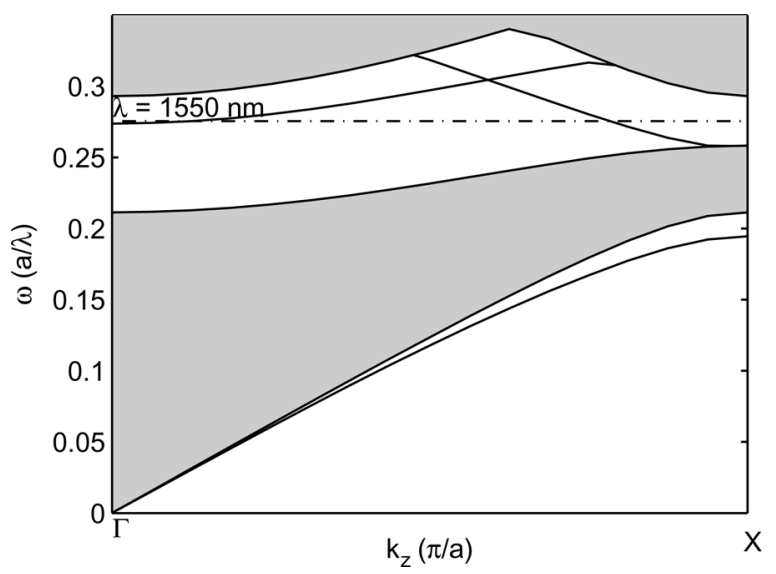

Fig. 3. Projected band diagram of a pillar-based photonic crystal waveguide in a polymer layer stack, where $a=427 \mathrm{~nm}, r=107 \mathrm{~nm}$ and $r_{\mathrm{d}}=155 \mathrm{~nm}$.

On the other hand, the even mode has a large and constant slope around $\lambda=1550 \mathrm{~nm}$. This regime is favorable for waveguiding purposes, since the group velocity is high and the wavelength dispersion is low over a considerable bandwidth. By properly tapering the classical ridge waveguide to the photonic crystal waveguide, we are able to excite only the even mode. The frequency range over which the slope of the even guided mode is large runs from $0.265(a / \lambda)$ to $0.290(a / \lambda)$, corresponding to a wavelength range from 1.47-1.61 $\mu \mathrm{m}$. This is sufficient band width for practical applications in the EDFA window.

\section{OUT-OF-PlanE CONFINEMENT}

In this section, we will investigate the possibility of creating confinement between the III-V pillars by implementing a polymer layer stack [10]. Although polymers are available in a relatively wide range of refractive index, our choice is limited by further demands such as transmission and absorption spectra, chemical stability etc. The polymers that we selected have indices $n_{1, \text { core }}=1.64$ and $n_{1, \text { clad }}=1.38$ at a wavelength $\lambda=1550 \mathrm{~nm}$. With these refractive indices, we will optimize the layer thickness of the high-index polymer and the etch depth of the pillars. The optimization is done with 2-D calculations of a vertical cross section through the center of the $\mathrm{PhC}$ waveguide pillars [11]. This structure effectively represents a 1-D grating. The simulations are intended to investigate the improvement that can be obtained by optimizing the polymer waveguide. Finally, the optimized polymer layer stack is used in the analysis of the Bloch modes of the grating. These Bloch modes are linked to the band diagrams of the photonic crystal waveguides.

\section{A. Grating Simulations}

The simulated structure is a 1-D periodic grating of semiconductor pillars interrupted by low-index regions consisting of either air, a polymer layer stack or a uniform polymer with a refractive index that is equal to the effective refractive index of the polymer layer stack.

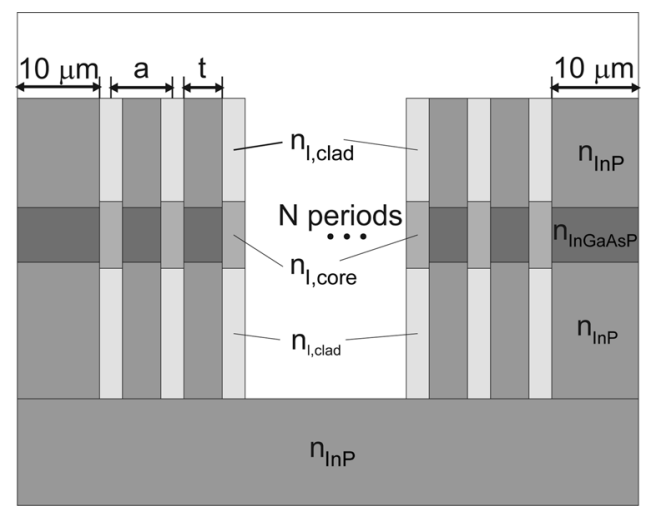

Fig. 4. Schematic structure of the 1-D grating used in the simulations.

The structure that is simulated, is schematically drawn in Fig. 4. It consists of an input waveguide that is $10 \mu \mathrm{m}$ long, followed by a one-dimensional grating of $N$ periods and an output waveguide of again $10 \mu \mathrm{m}$ length. One period of the grating consists of a high-index semiconductor region with a length of $t=310 \mathrm{~nm}$ and a lattice constant of $a=427 \mathrm{~nm}$. The choice for the geometrical parameters is based on the photonic crystal waveguide parameters for pillars in a polymer stack, also for the pillars in air and pillars in a uniform polymer layer.

Since we aim for the integration of PhC-based components in classical PICs, we are restricted by the semiconductor layer stack consisting of an InP substrate, an InGaAsP core layer of $500-\mathrm{nm}$ thickness and an InP top cladding layer of $1 \mu \mathrm{m}$. In these simulations, the center lines of the semiconductor and polymer cores are always at the same height. The refractive indices of the semiconductor materials are $n_{\mathrm{InP}}=3.169$ and $n_{\mathrm{InGaAsP}}=$ 3.364 at a wavelength $\lambda=1550 \mathrm{~nm}$.

To calculate the transmission and the reflection of the grating, an eigenmode expansion method is used [12]. At the input waveguide, the fundamental guided TM mode is excited. $T$ is the transmission from that mode to the fundamental guided TM mode at the output waveguide. $R$ is the reflection to the fundamental TM mode at the input waveguide. From these two coefficients, the loss is calculated as $L=1-T-R$.

Fig. 5 shows the transmission, reflection and loss of the grating for varying polymer core thickness, where the etch depth is initially taken to penetrate the full semiconductor stack. The reflection is nearly constant over the full range of polymer core thickness, and it is in agreement with the Fresnel reflection at the interface. If the polymer core thickness increases from $0.2 \mu \mathrm{m}$ to $0.8 \mu \mathrm{m}$, the coupling loss steadily decreases because the mode profile of the polymer waveguide gets more confined to its core layer. This provides a better overlap with the mode of the InP/InGaAsP/InP waveguide. Increasing this thickness even more, however, reduces the coupling again, because the mode in the polymer waveguide starts to scale with the thickness. For polymer core thickness $>1.0 \mu \mathrm{m}$ the polymer waveguide ceases to be single-mode, and light can be coupled into higher order modes too. The second minimum in the transmission around $d_{\mathrm{l} \text {,core }}=1.7 \mu \mathrm{m}$ is due to mode matching to a higher order mode. Since these higher order modes destroy the in-plane photonic crystal properties, 


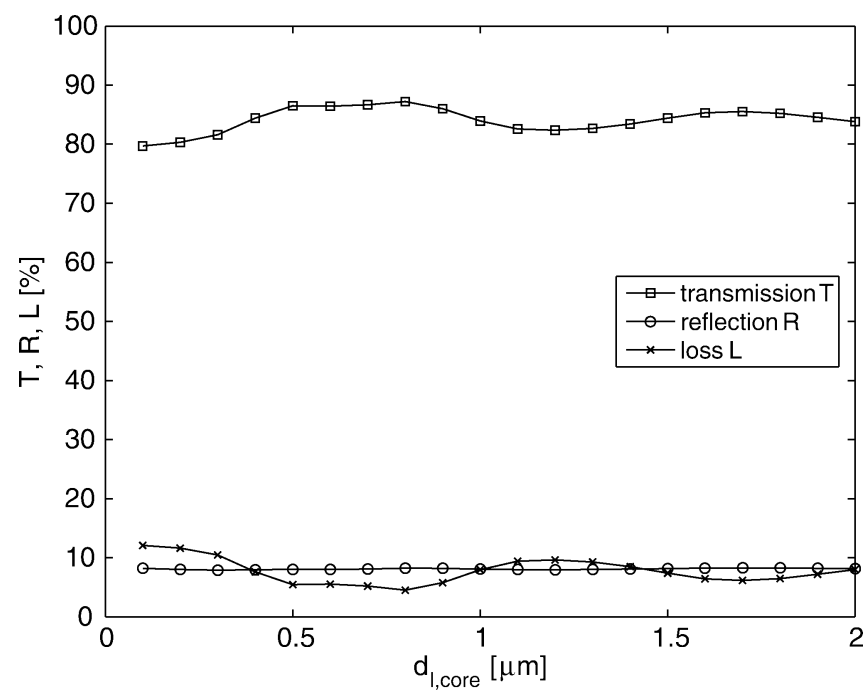

Fig. 5. Transmission, reflection and loss as a function of polymer core thickness for a 1-D periodic structure. All values are averaged over 30 to 60 periods to average out oscillations that are due to the finite grating length.

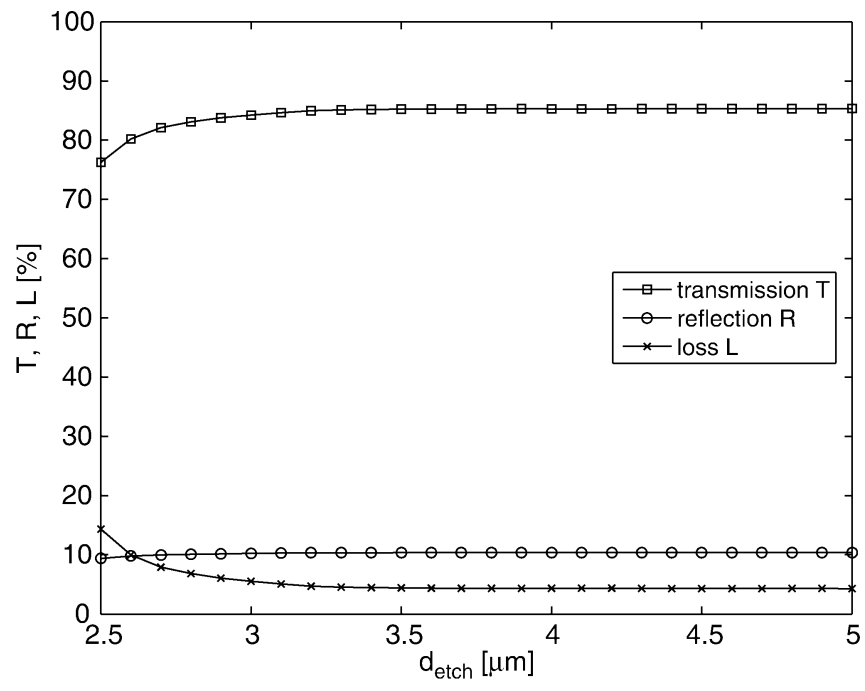

Fig. 6. Transmission, reflection and loss as a function of etch depth for a grating with a polymer layer stack with $d_{1, \text { core }}=800 \mathrm{~nm}$.

multimode operation should be avoided. The optimal polymer core layer thickness is thus $d_{1, \text { core }}=0.8 \mu \mathrm{m}$, resulting in $5 \%$ coupling loss.

Fig. 6 shows the calculated reflection, transmission and loss of the grating with the polymer layer stack as a function of the etch depth. For etch depth $>3 \mu \mathrm{m}$, the loss is $\sim 5 \%$, and this value increases for smaller etch depth.

Fig. 7 shows the losses in the three cases in $\mathrm{dB}$ as a function of the number of periods $N$ of the grating. For a grating of sufficient length, the losses consist of a contribution that linearly increases with the length and of a contribution that is constant regardless of the length. From the linear fits, we can get an estimation of the propagation losses in the transmission window of the grating. For the pillars in air, the estimation obtained in this way is $\sim 109 \mathrm{~dB} / \mathrm{cm}$. The losses are reduced to $\sim 70 \mathrm{~dB} / \mathrm{cm}$ if a uniform polymer is introduced between the pillars. By applying

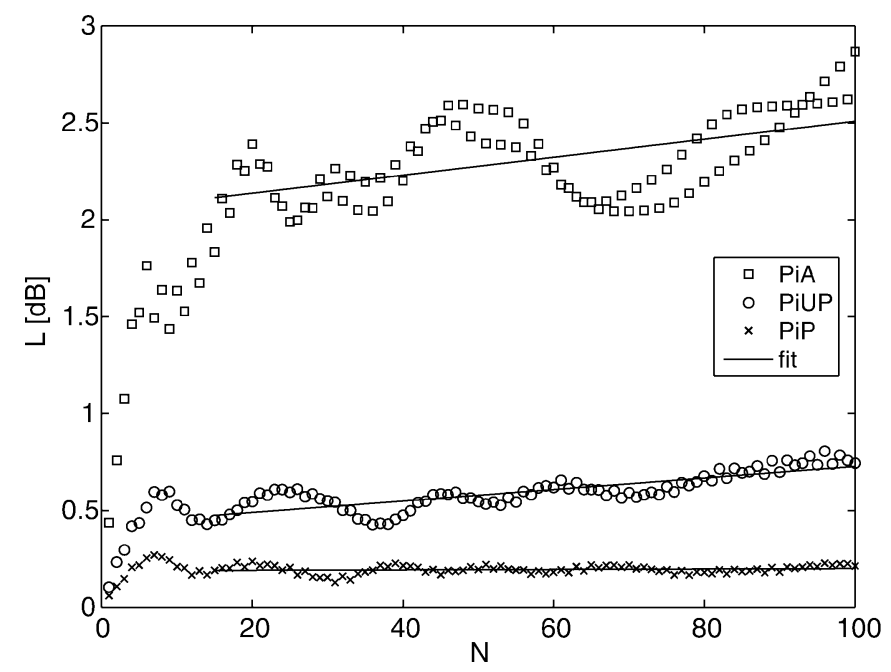

Fig. 7. Loss as function of the total number of periods $N$ of the grating for three different fillings between the semiconductor pillars: for pillars in air ( $\mathrm{PiA})$, pillars in a uniform polymer with $n=n_{1 \text {,eff }}$ (PiUP) and pillars in the optimized polymer layer stack (PiP). The etch depth is $3 \mu \mathrm{m}$.

the optimized polymer layer stack, the estimated losses of the 1 -D grating are as low as $\sim 3 \mathrm{~dB} / \mathrm{cm}$.

The constant fractions of the losses in Fig. 7 are due to the transition from a slab waveguide mode to a grating Bloch mode. These transition losses are $\sim 2.0 \mathrm{~dB}$ for pillars in air, decreasing to $\sim 0.4 \mathrm{~dB}$ for pillars in a uniform polymer layer. This reduction in transition losses can be contributed to the reduction in diffraction angle. The transition loss is even smaller for pillars with the optimized polymer layer stack, $\sim 0.2 \mathrm{~dB}$. This loss is due to the transitions between the different types of modes in the ridge waveguide and in the grating, and it corresponds to the 5\% coupling loss that was found in Fig. 5.

From the estimated propagation losses it becomes clear that a reduction in index contrast between pillars and surrounding medium reduces the losses. Presumably, this is because the diffraction angle is smaller, so a larger amount of the light is recaptured by the next pillar. However, the fact that optical guiding is introduced between the pillars reduces the losses in $\mathrm{dB}$ by one to two orders of magnitude. Although the simulated structure is a 2-D representation of a 3-D situation, the results clearly indicate that in pillar-based photonic crystals a huge reduction in propagation losses can be obtained by implementation of a polymer layer stack. The propagation loss in an actual pillar-based photonic crystal waveguide will be larger, as the lower limit of a classical ridge waveguide in InP technology is in the order of $1 \mathrm{~dB} / \mathrm{cm}$. The lower limit is imposed by absorption and sidewall roughness of the ridge waveguide. Furthermore, in-plane losses, e.g. because of imperfect definition of the periodic structure, will also contribute to the total losses of a 3-D photonic crystal waveguide.

\section{B. Bloch Mode Analysis}

In the previous section, we have shown that the propagation losses can be significantly reduced by implementing an optimized polymer layer stack between the semiconductor layer stack. In this section, we will explain the physical principles behind these extremely low propagation losses. 


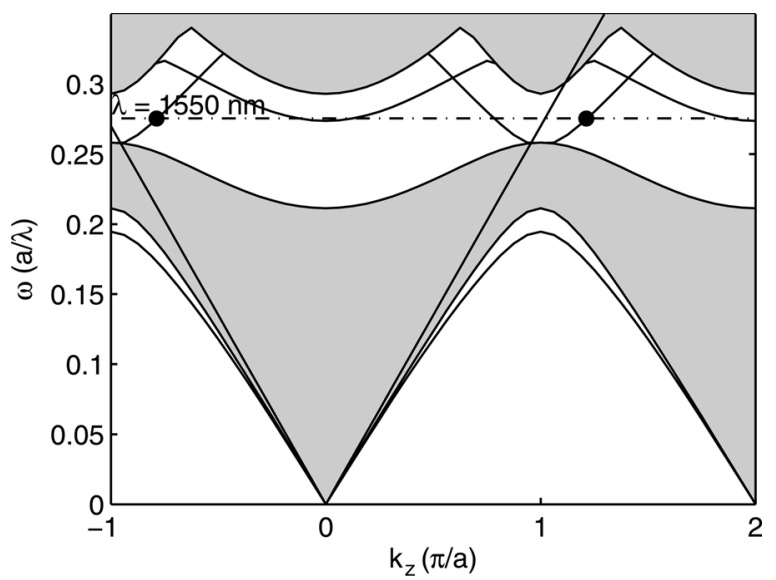

Fig. 8. Projected band diagram of a $\mathrm{PhC}$ waveguide, extended over one-and-ahalf Brillouin zone. It corresponds to the $\mathrm{PhC}$ waveguide of pillars in polymers, where $r=107 \mathrm{~nm}, a=427 \mathrm{~nm}$, and $r_{\mathrm{d}}=155 \mathrm{~nm}$. The dots indicate the Fourier components of the Bloch mode in subsequent Brillouin zones.

In fact, the band diagram of the first Brillouin zone of the 1-D grating is repeated infinitely in positive and in negative direction of k. Fig. 8 shows the projected band diagram of a $\mathrm{PhC}$ waveguide, extended over one-and-a-half Brillouin zone. At the frequency corresponding to $\lambda=1550 \mathrm{~nm}$, the Bloch mode consists of components from all Brillouin zones. The field of this Bloch mode at a certain instance in time can be written as

$$
E(y, z)=\sum_{m=-\infty}^{\infty} c_{m}(y) e^{j\left(k_{z}+2 \pi m / a\right) z}
$$

However, not all spatial frequency components are equally strong represented in a Bloch mode. By Fourier transforming the field of the actual excited Bloch mode, the relative strengths of the spatial frequency components can be found [13].

To this end, we investigate the guided Bloch mode in the 1-D grating simulated in the previous section. By taking the 2-D Fourier transform of the field profile, we obtain the relative strengths of its spatial frequencies $k_{z}$ (in propagation direction) and $k_{y}$ (out-of-plane).

The complex field amplitude of the Bloch mode that is excited in a 1-D grating is calculated using a finite-difference time domain (FDTD) method [8]. The parameters used for the lengths of semiconductor and low-index sections in the grating are the same as in the previous section. The grating length is kept at 50 periods. PMLs are placed at the boundaries of the calculation window. The input waveguide is excited with the fundamental guided TM mode in continuous-wave operation at a wavelength $\lambda=1550 \mathrm{~nm}$. Fig. 9 shows the field amplitudes after $500 \mathrm{fs}$ (when the field is stable) for the three different cases: pillars in air, pillars in a uniform polymer layer, and pillars in the optimized polymer layer stack. From the figures it is qualitatively visible that for the pillars in air, the radiation of light to the air and to the substrate is higher than for the other two cases. The case where the optimized polymer layer stack was applied seems to have the least radiation.

Taking the Fourier transforms of the field amplitudes provides us with the relative strengths of the spatial frequencies. In the field plots of Fig. 9, we encounter three regions; the field at the

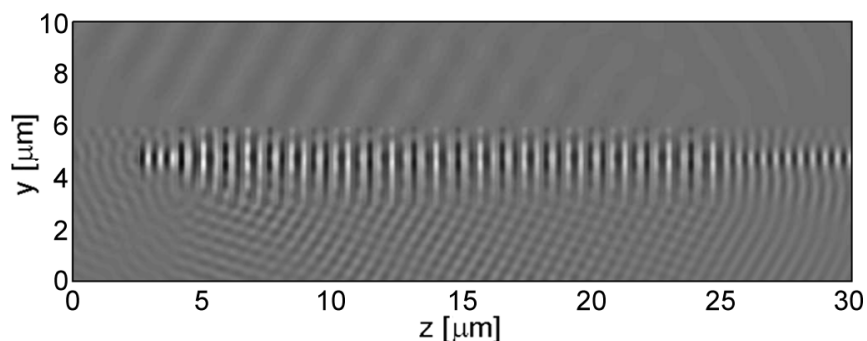

(a)

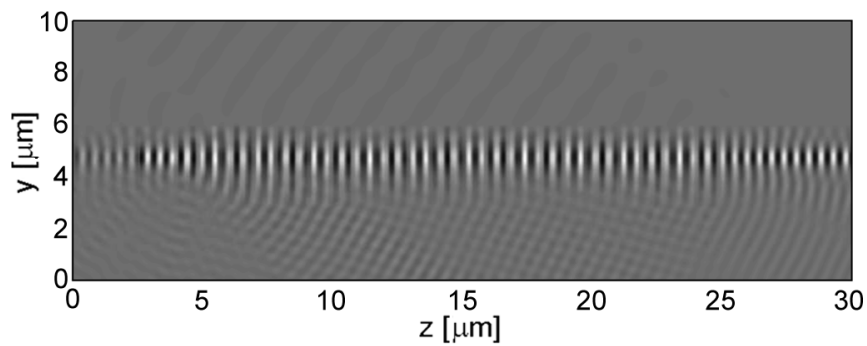

(b)

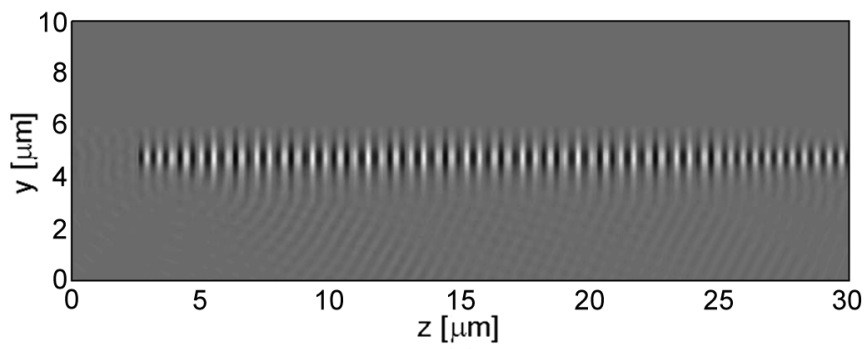

(c)

Fig. 9. Field amplitudes in a 50-period 1-D grating after 500 fs for (a) pillars in air, (b) pillars in a uniform polymer layer, and (c) pillars in the optimized layer stack.

input an output ridge waveguides, the field inside the grating and the fields at the transitions between both types of waveguides. From Fig. 7, we learned that the transition from a ridge waveguide mode to a grating Bloch mode spans about ten periods. Since we are only interested in the Fourier components of the grating Bloch modes, we do not take into account the first and the last ten grating periods when taking the Fourier transforms. The field over the center 30 periods of the grating is Fourier transformed to find the strengths of the spatial frequency components.

The results of the 2-D Fourier transforms of the three cases are shown in Fig. 10. Both $k_{z}$ and $k_{y}$ are expressed in $(\pi / a)$. The light lines of air and InP at $\lambda=1550 \mathrm{~nm}$ are plotted in the graphs as semicircles, where the light line of air is only represented for positive $k_{y}$ and that of InP is only represented for negative $k_{y}$. The full circle represents a medium with a refractive index equal to the average refractive index $n_{\mathrm{av}}$ of the InP grating claddings

$$
n_{\mathrm{av}}=\frac{n_{\mathrm{InP}} \cdot t+n_{\mathrm{l}, \mathrm{clad}} \cdot(a-t)}{a} .
$$

This light cone only approximates the $|\mathbf{k}|$-vectors of cladding modes, because the claddings are no homogeneous media with this average refractive index. All modes that are excited in the grating either belong to the guided Bloch mode without an 




(a)

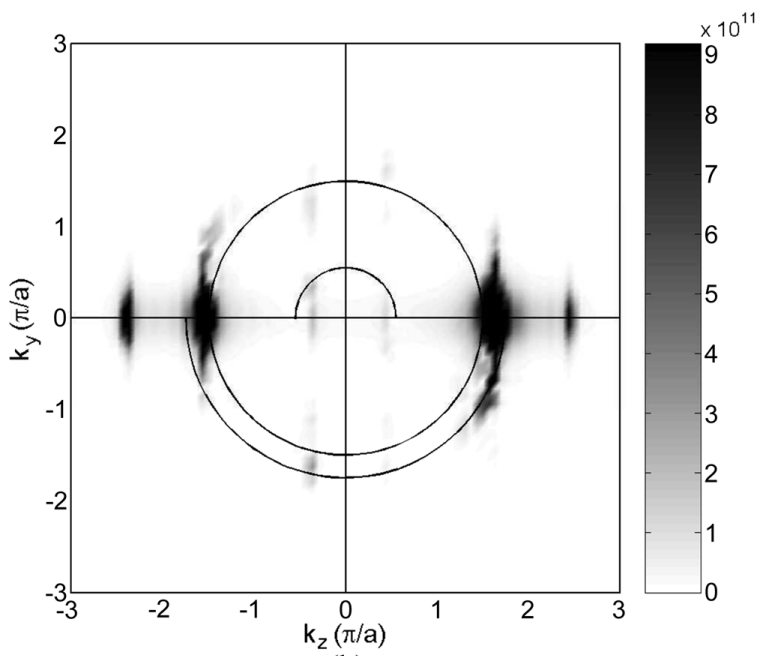

(b)

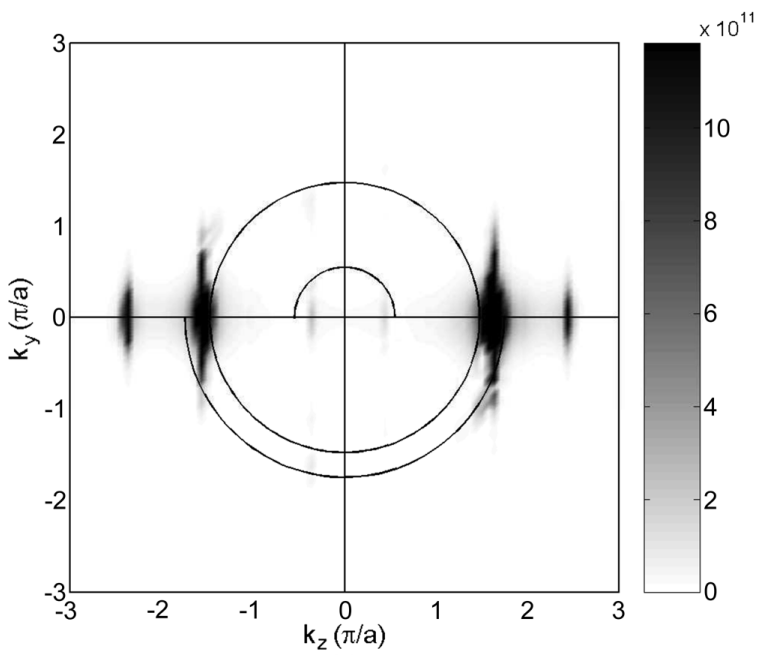

(c)

Fig. 10. Fourier transform of the field amplitudes over the center 30 periods in a 50-period 1-D grating after 500 fs for (a) pillars in air, (b) pillars in a uniform polymer layer, and (c) pillars in the optimized layer stack. The grayscale is adapted to improve the visibility of the relevant spectral peaks.

out-of-plane component (i.e., modes having only a $k_{z}$ component) or they are out-of-plane modes on one of the light lines



Fig. 11. Fourier spectrum along the line of constant $k_{z}$ belonging to the grating Bloch mode in the first Brillouin zone with $-\pi / a \leq k_{z} \leq 0$ for pillars in air (PiA), pillars in a uniform polymer (PiUP) and pillars in the optimized polymer layer stack (PiP).

belonging to the claddings, the air above or the InP below. The $k_{z}$ values of all modes are determined by the Bloch mode of the grating, and the $k_{y}$ values of the radiation modes follow from the light cones of the corresponding media. The peaks in the Fourier spectrum that are not on one of the light lines nor on the $k_{y}=0$ axis, are ghost peaks that are probably due to the finite step size in the FDTD calculation of the complex field amplitude.

From Fig. 10(a) we can see that the pillars in air have relatively strong radiation mode components. On the air light cone, a strong peak visualizes the radiation into the air, as we could see from the field plot in Fig. 9(a). On the InP light cone there are three peaks, and indeed in the field plot the InP substrate shows a more complicated radiation pattern with interfering plane waves. The presence of the peaks on the cladding light cone shows that coupling to the radiation modes is possible via intermediate coupling to the cladding modes. The cladding modes do not exactly coincide with the cladding light cone due to the fact that this light cone is an approximation based on the weighted average refractive index of the claddings.

For the pillars in a uniform polymer layer, for which the spatial Fourier transform is given in Fig. 10(b), the strengths of the components in the first Brillouin zone are significantly reduced, although they are still present (note the difference in gray scale in the Fourier spectrum). Finally, Fig. 10(c) shows the Fourier spectrum of the pillars with the optimized polymer layer stack. The peaks on the light cones have nearly vanished from the spectrum.

The peaks belonging to the $k_{z}$ in the first Brillouin zone have the largest contribution to the out-of-plane losses. In Fig. 11 the relative strengths of these peaks are compared. The curves are normalized to the peak belonging to the guided Bloch mode, i.e., the one at $k_{y}=0$. All three curves belong to the spatial frequency of the Bloch mode with $-\pi / a \leq k_{z} \leq 0$ (the peaks in the positive half of the first Brillouin zone are ghost peaks). The actual $k_{z}$ value is slightly different in each case, as the Bloch mode depends on the refractive indices of the grating. In the 
graph, the reduction in the coupling to out-of-plane radiation modes is clearly visible.

Apart from the coupling to radiation modes by intermediate coupling to the cladding modes, there is also a coupling mechanism that is probably due to the penetration of the evanescent tails of the guided Bloch mode into the InP substrate. This peak is the one on the InP light cone with $\pi / a \leq k_{z} \leq 2 \pi / a$. Its $|\mathbf{k}|$ value is too large to couple to the cladding modes. The implementation of the polymer layer stack reduces this out-of-plane loss mechanism because of the smaller diffraction angle. By increasing the etch depth further into the InP substrate, the direct coupling to the InP substrate via the evanescent tails will vanish completely.

The polymer layer stack maximizes the coupling to the Fourier components with high in-plane k-vectors, thereby minimizing the coupling to $\mathrm{k}$-vectors with a small $k_{z}$ that are able to couple to the radiation modes. Recalling the band diagram in Fig. 8, the Bloch components above the light line are strongly reduced and effectively, we excite a Bloch mode with only components outside the light cone. This mechanism enables us to design pillar-based $\mathrm{PhC}$ components with low propagation losses.

\section{CONCLUSION AND DisCUSSION}

It has been shown that line defects in 2-D pillar-based photonic crystals can act as low-loss waveguides. The photonic band gap properties of the crystal induce the in-plane confinement, whereas out-of-plane confinement is due to total internal reflection. It has been shown that the implementation of a polymer layer stack between the pillars can significantly reduce the propagation losses of $\mathrm{PhC}$ waveguides. In order to understand the low propagation losses in pillar-based photonic crystals, the physical principles of the Bloch modes have been investigated. From this it becomes clear that the polymer layer stack minimizes the coupling to Bloch mode components with low $k_{z}$-values inside the light cone.

The fabrication technology for the integration of the pillarbased photonic crystals in classical PICs has been developed [14]. The implementation of the polymer layer stack is reported in [15]. Because of the predicted low losses and the additional technological advantages, pillar-based photonic crystals form a strong platform for integration of a new series of devices in classical PICs. Currently, the realization of photonic crystal waveguides with an implemented polymer layer stack is in progress.

\section{REFERENCES}

[1] Y. Akahane, T. Asano, B.-S. Song, and S. Noda, "High-Q photonic nanocavity in a two-dimensional photonic crystal," Nature, vol. 425, pp. 944-947, Oct. 2003.

[2] H. Takano, Y. Akahane, T. Asano, and S. Noda, "In-plane-type channel drop filter in a two-dimensional photonic crystal slab," Appl. Phys. Lett., vol. 84, no. 13, pp. 2226-2228, 2004.

[3] H. T. Hattori, I. McKerracher, H. H. Tan, C. Jagadish, and R. D. L. Rue, "In-plane coupling of light from InP-based photonic crystal band-edge lasers into single-mode waveguides," IEEE J. Quantum Electron., vol. 43, no. 3, pp. 279-286, Mar. 2007.
[4] V.Zabelin, L. Dunbar, N. L. Thomas, R. Houdré, M. Kotlyar, L. O'Faolain, and T. F. Krauss, "Self-collimating photonic crystal polarization beam splitter,' Opt. Lett., vol. 32, pp. 530-532, Mar. 2007.

[5] L. Wu, M. Mazilu, T. Karle, and T. F. Krauss, "Superprism phenomena in planar photonic crystals," IEEE J. Quantum Electron., vol. 38, pp. 915-918, Jul. 2002.

[6] H.-G. Park, S.-H. Kim, M.-K. Seo, Y.-G. Ju, S.-B. Kim, and Y.-H. Lee, "Characteristics of electrically driven two-dimensional photonic crystal lasers," IEEE J. Quantum Electron., vol. 41, no. 9, pp. 1131-1141, Sep. 2005.

[7] R. van der Heijden, C. Carlström, J. Snijders, R. van der Heijden, F. Karouta, R. Nötzel, H. Salemink, B. Kjellander, C. Bastiaansen, D. Broer, and E. van der Drift, "InP based two-dimensional photonic crystals filled with polymer," Appl. Phys. Lett., vol. 88, 2006, 161112.

[8] Photon Design, CrystalWave. [Online]. Available: http://www.photond.com

[9] J. D. Joannopoulos, R. D. Meade, and J. N. Winn, Photonic Crystals: Molding the Flow of Light. Princeton, NJ: Princeton Univ. Press, 1995.

[10] J. Čtyroký, "Photonic band gap structures in planar waveguides," $J$. Opt. Soc. Amer. A, vol. 18, pp. 435-441, Feb. 2001.

[11] W. Bogaerts, P. Bienstman, D. Taillaert, R. Baets, and D. de Zutter, "Out-of-plane scattering in 1-D photonic crystal slabs," Opt. Quantum Electron., vol. 34, pp. 195-203, 2002.

[12] P. Bienstman, CAMFR. [Online]. Available: http://camfr.sourceforge.net

[13] W. Kuang, W. J. Kim, A. Mock, and J. O'Brien, "Propagation loss of line-defect photonic crystal slab waveguides," IEEE J. Sel. Topics Quantum Electron., vol. 12, no. 11, pp. 1183-1195, Nov. 2006.

[14] A. Kok, E. Geluk, B. Docter, J. van der Tol, R. Nötzel, M. Smit, and R. Baets, "Transmission of pillar-based photonic crystal waveguides in InP technology," Appl. Phys. Lett., vol. 91, Nov. 2007, 201109.

[15] A. Kok, E. Geluk, M. Sander-Jochem, J. van der Tol, Y. Oei, and M. Smit, "Two-dimensional photonic crystals based on InP rods," in Proc. IEEE/LEOS Symp. (Benelux Chapter), Mons, Belgium, 2005, pp. 273-276.



Abigaël A. M. Kok (S'01-M'08) was born in Heinkenszand, The Netherlands, in 1979. She received the M.Sc. degree in applied physics from Twente University, Enschede, The Netherlands, in 2002 and the Ph.D. degree in photonic crystals in InP-based optical integrated circuits from the Opto-Electronic Devices Group, Faculty of Electrical Engineering, Eindhoven University of Technology, Eindhoven, The Netherlands, in 2008.

After the M.Sc. degree, she continued her research for six months at the Lightwave Devices Group, Twente University, as a Junior Researcher. Currently, she is with the FEI Company (The Netherlands), specializing in transmission electron microscopy

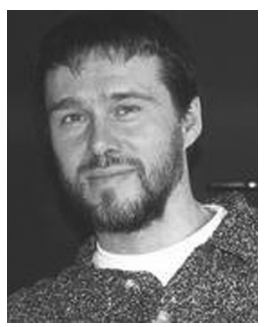

Jos J. G. M. van der Tol was born in Alphen a/d Rijn, The Netherlands, in 1956. He received the M.Sc. and Ph.D. degrees in physics from the State University of Leiden, Leiden, The Netherlands, in 1979 and 1985, respectively.

In 1985, he joined KPN Research, where he became involved in research on integrated optical components for use in telecommunication networks. His research interest in this field have covered modeling of waveguides, design of electro-optical devices on lithium niobate, and their fabrication. Furthermore, he has been working on guided wave components on III-V semiconductor materials. He has also been active in the field of optical networks, focusing on survivability, introduction scenarios, and management issues. Since July 1999, he has been an Associate Professor at the University of Technology Eindhoven, Eindhoven, The Netherlands, where his research interests include opto-electronic integration, polarization issues, and photonic crystals. He is the author or coauthor of more than 130 publications in the fields of integrated optics and optical networks and has 25 patent applications to his name. 


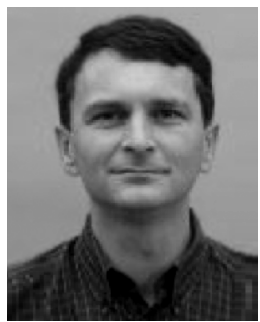

Roel Baets (M'88-SM'96-F'07) received the electrical engineering degree from Ghent University, Ghent, Belgium, in 1980, the M.Sc. degree in electrical engineering from Stanford University, Stanford, CA, in 1981, and the Ph.D. degree from Ghent University in 1984.

He joined the Department of Information Technology (INTEC), Ghent University, in 1981, and since 1989, he has been a Professor with the engineering faculty. From 1990 to 1994, he was a Part-Time Professor with the Technical University of Delft, Delft, The Netherlands. He has mainly worked in the field of photonic components. With about 300 publications and conference papers as well as about ten patents, he has made contributions to the design and fabrication of III-V semiconductor laser diodes, passive guided-wave devices, photonic integrated circuits, and micro-optic components. He currently leads the Photonics Group at the INTEC, Ghent University, which is an associated laboratory of the Interuniversity Microelectronics Center, working on integrated photonic devices for optical communication, optical interconnect, and optical sensing.

Dr. Baets is a member of the Optical Society of America, the IEEE Lasers and Electro-Optics Society (where he was formerly a Chairman of the Benelux Chapter from 1999 to 2001 and is currently a member of the Board of Governors, the International Society for Optical Engineers), and the Flemish Engineers Association. He has been member of the program committees of the Optical Fiber Communications Conference, the European Conference on Optical Communication, the IEEE Semiconductor Laser Conference, the European Solid-State Device Research Conference, the Conference on Lasers and Electro-Optics, Europe, and the European Conference on Integrated Optics.

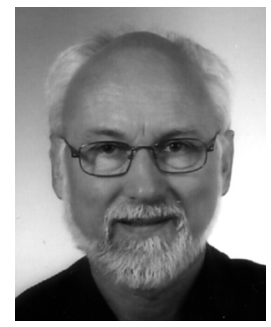

Meint K. Smit (M'00-SM'01-F'03) received the Electrical Engineering and Ph.D. degrees (both with honors) from the Delft University of Technology, Delft, The Netherlands, in 1974 and 1991, respectively.

In 1974, he started work on radar and radar remote sensing. He joined the Delft University of Technology in 1976. He switched to optical communications in 1981. His research field is integrated optics. He became Leader of the Photonic Integrated Circuits Group, Delft University, in 1994. He was appointed Professor in 1998. In 2002, he moved with his group to the Technical University of Eindhoven, Eindhoven, The Netherlands, where he is currently the Leader of the Opto-Electronic Devices Group, COBRA Research Institute.

Dr. Smit received the LEOS Technical Achievement Award in 1997 for his invention of the Arrayed Waveguide Grating . In 2002, he was appointed LEOS Fellow for contributions in the field of Opto-Electronic Integration. 